\title{
Geoinclusão: Sistemas de Informação Geográfica e Crowdthinking
} Geoinclusion: Geographical Information Systems and Crowdthinking

\author{
Leonardo Oliveira Muniz \\ Universidade Federal do Rio de Janeiro, Brasil \\ leonardomuniz@poli.ufri.br
}

Tiago Badre Marino

Universidade Federal Rural do Rio de Janeiro, Brasil

tiagomarino@hotmail.com

\author{
Jorge Xavier da Silva \\ Universidade Federal do Rio de Janeiro, Brasil \\ xavier.lageop@gmail.com
}

\begin{abstract}
This article aims to disseminate the concept of "geoinclusion" for the iberoamerican scientific community. This concept was created in 2007 by the 'GIS Laboratory' of the Federal University of Rio de Janeiro (Brazil) to operationalize the "crowdthinking" through Geographic Information Systems (specially the web and mobile versions). Ever since, it was tested by educational, health, safety and environment sectors and the result was the creation and continuous improvement of the web plataform VICON.SAGA. The paper will demonstrate its four fundamental principles of knowledge construction: decentralization, authenticity, integration and resilience.
\end{abstract}

Keywords: Geoinclusion; Geographical Information Systems; Crowdthinking; VICON.SAGA.

\section{Introdução}

O uso de geotecnologias tem cada vez mais se popularizado. A geolocalização por dispositivos móveis dá ao usuário as melhores rotas para seu dia, a proximidade de pessoas a ele conectadas, os estabelecimentos comerciais ao entorno, as condições ambientais esperadas para um determinado local e horário. Portanto, o que emerge a partir do público leigo (não-especializado) a cada check-in e share é a geoinformação do cotidiano, de maneira distribuída, integrada e resiliente. Isto é bem demonstrado por Xavier da Silva (2011) e Cereda Jr. (2015).

A partir de suas observações factuais, dentro do atual contexto tecnológico da informação (Big Data, Data Mining, Business Intelligence, Internet of Things, entre outros) e político (democratização da informação, seja ela geográfica ou não), são mapeadas opiniões, ideias, projetos novos e inovadores, iniciativas de instâncias públicas ou privadas. Analogamente ao crowdthinking, portanto, as geotecnologias apresentam um potencial engajamento entre pessoas dos mais diversos setores sociais e de trabalho inseridas em seus espaços virtuais-reais percebidos, como são as chamadas redes sociais (Facebook, Twitter, Linkedln).

Um conceito dentro do ramo das geotecnologias, mais precisamente dos SIGs Web e Apps (Mobile), que surge com esta proposta já há cerca de 10 anos, é a Geoinclusão ou Inclusão Geográfica/Ambiental (Xavier da Silva, 2008). Para o autor do conceito, há um prosseguimento lógico das inclusões digital, social e geográfica a partir do momento em que tecnologias de uso difuso e intuitivo permitem ao público leigo (não especializado) a orientação ou a criação de mapas para o dia-a-dia, inclusive no sentido pedagógico da percepção e interação espaciais, como será visto mais adiante. O interesse maior, no entanto, é o de familiarizar o público com as ferramentas disponíveis para que, a médio prazo, haja a noção e o uso não apenas reativo destes dados; avançando na construção de estruturas de controle, análise, planejamento e gestão autônomos (Muniz da Silva, 2013).

A proposta deste artigo é demonstrar este conceito em operação, com casos reais, aplicados, em que SIG e crowdthinking caminharam juntos para uma construção social e empreendedora.

Como sugerido desde os primeiros autores sobre SIGs e Geoprocessamento, o referencial geográfico se faz imprescindível para se obter ganho de conhecimento acerca da realidade ambiental nas mais diversas escalas. Hoje, isto é mais claro, pois, a partir do local (usuário e dispositivos), grandes massas de dados são geradas permitindo: a) a representação de vizinhanças, bairros, municípios, estados, lugares, etc. e; b) a manipulação e transformação desta massa em informação relevante para o apoio de decisões no que tange à participação cidadã no fazer ambiental, no engajar-se, nos desenvolvimentos pessoal e comunitário.

A hipótese deste estudo é de que o crowdthinking aplicado às geotecnologias, como ocorre com a geoinclusão, potencializa ganhos, oportunidades e, além disto, minimizando custos e ampliando comunidades colaborativas na construção de projetos.

\section{Referencial teórico}

Usualmente, o termo "geotecnologias" é utilizado para se referir ao conjunto de campos técnico-científicos que permitem a geração e manutenção de bases de dados e/ou a extração de informação geográficos, isto é, atribuídos de referencial geográfico (sistema de coordenadas ou localização por endereços). Esta é uma definição ampliada e 
pragmática extraída de Xavier da Silva (2009). O autor nos alerta sobre as confusões conceituais decorrentes de um boom de termos que, antes mais restritos às universidades, tomam proporções mais globalizantes. Ou seja, como já introduzido, há, hoje, uma nova tendência de aproximação entre Geografia e Tecnologia para além do uso especializado. A geolocalização por dispositivos móveis amplamente difundidos exemplifica isto fazendo Cereda Jr. (2015) cunhar o termo "Geografia das Coisas", "que amplia o horizonte da Internet das Coisas e confere humanidade a ela":

"A chamada "Internet das Coisas" (em inglês, Internet of Things - IoT), termo cunhado por Kevin Ashton, do MIT (Instituto De Tecnologia De Massachusetts) em 1999, não tem um conceito claro ou único, mas podemos entendê-la como outra forma de descrever uma rede de dispositivos, pessoas ou equipamentos interconectados. [...] Vivemos a Geografia das Coisas, que amplia o horizonte da Internet das Coisas e confere humanidade a ela".

Apesar de posterior à geoinclusão, o termo "Geografia das Coisas" se faz relevante para sua compreensão, uma vez que bem descreve o contexto tecnológico social onde a geoinclusão encontra oportunidades para sua manifestação difusa. A partir do momento em que pessoas, equipamentos e recursos tem identificadas suas referências espaciais, elas são integradas em plataformas que permitem a visualização de cenários como não antes percebidas. Materializa-se o previsto pelo "pai do SIG" Roger Tomlinson (1962): "quando você descobre a Geografia, você ganha um novo par de olhos" (op. cit.).

Com esta noção, uma primeira definição para a inclusão geográfica encontra-se na seguinte pergunta: "Qual o caminho para a liberação do potencial de criação de soluções que usem as idiossincrasias locais e, principalmente, estejam respaldadas no conhecimento da distribuição espacial dos problemas efetivos de cada localidade?" (Xavier da Silva, 2008, pg. 3). Com as pessoas e recursos interconectados, dados relativos à experiência local nutrem modelos digitais do ambiente, relatando interesses comuns, oportunidades de coesão social e soluções de problemas. Em um sentido empreendedor, a inclusão de dados sobre a realidade em modelos digitais do ambiente clarifica oportunidades de engajamento em projetos de melhorias para grupos sociais que representam, portanto, coletividades.

Clarificando as diferenças: a geoinclusão pode ser definida como sendo a autopropulsionada inclusão digital, social e geográfica através da criação e atualização descentralizada de bases de dados distribuídos pela periferia do sistema (Xavier da Silva, 2011). A Geografia das Coisas são os condicionantes ideias para essa inclusão, que é um processo com diferentes níveis de participação possíveis. Ela representa a rede distruibuída de pessoas, dispositivos e recursos que facilitam a execução da geoinclusão.

Finalmente, chega-se ao crowdthinking, que, apesar de pouco difuso em periódicos científicos, apresenta uma definição consistente entre ativistas sociais pelo mundo
(Carroll, 2015): é uma rede de pensamento colaborativo que permite a representação do conhecimento e de ideias para o futuro das pessoas $\mathrm{e}(\mathrm{m})$ suas interações espaciais: melhoria das relações entre pessoas, entre pessoa-equipamento, equipamento-equipamento, dando inteligência ao funcionamento de cidades, por exemplo.

Como a geoinclusão pode operacionalizar esta transformação será visto no próximo item, que apresenta a estrutura metodológica lócus da inclusão geográfica: os exaustivamente mencionados SIGs - Sistemas de Informação Geográfica.

\section{Proposta metodológica}

Os Sistemas de Informação Geográfica (SIGs, tradução do inglês para Geographical Information Systems - GIS) são geralmente definidos a partir de suas funções: "um conjunto integrado de hardware e software para a aquisição, armazenamento, estruturação, manipulação, análise e exibição gráfica de dados espacialmente referenciados pelas coordenadas geográficas" (Aronoff, 1989). São ferramentas computacionais que, portanto, facilitam o geoprocessamento de dados, isto é, a transformação de dados em informação relevante a partir de sua inclusão em modelos digitais do ambiente (representação computacional do espaço geográfico), análises e sínteses que propiciam o apoio a decisão referente aos recursos disponíveis então espacializados (Xavier da Silva, 2009). Baseado nisto, a metodologia que aqui se adota e se apresenta para a comunidade científica define como material um SIG e como base teórico-metodológica o geoprocessamento.

Representando a Geografia das Coisas, a situação que aqui se busca analisar é composta por dispositivos hardware distribuídos, interconectáveis via Internet e localizados através de coordenadas geográficas e endereços cadastrados sobre bases de dados de logradouros. A geoinclusão pode ser operada a partir de um input de dados diretamente sobre as bases georreferenciadas ou um output de dados convencionais previamente lançados com um atributo geográfico em sua estrutura, visível ou não para os usuários (a exemplo, tem-se os tweets e posts).

Estrutura-se: um SIG Web ou Mobile, alimentado a partir do uso de dispositivos móveis; a aquisição de dados de fontes diversas e distribuídas; a integração em um modelo digital do ambiente; por fim, a oportunidade e a execução de um projeto colaborativo. 


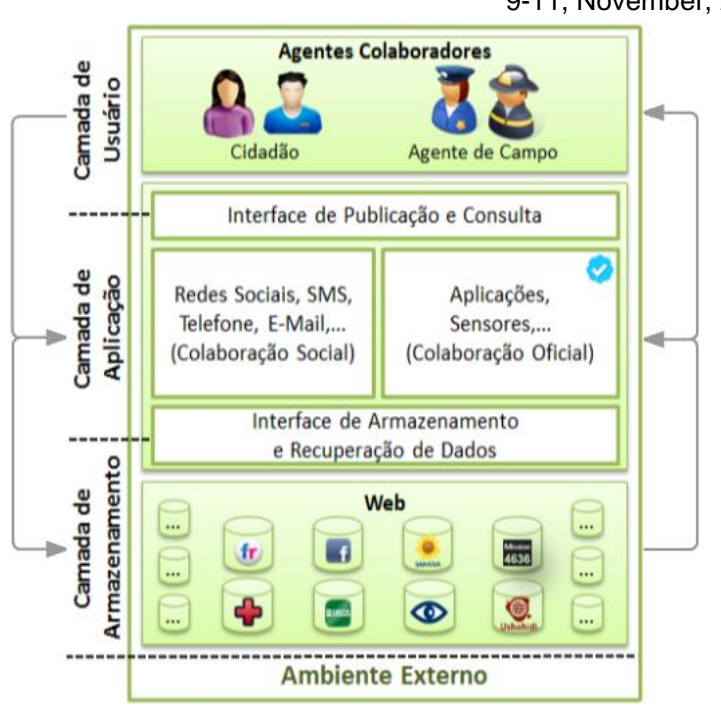

Figura 1: Modelo funcional do SIG Web e Mobile VICON (adaptado de MARINO, 2015, pg. 113). As setas indicam o fluxo de dados (à esquerda) e informações (à direita).

As etapas pelas quais passam os projetos são: 1) Vigilância - registro de ocorrências percebidas espaço-temporalmente; 2) Controle - acompanhamento e possibilidade de intervenção baseadas em dados; 3) Análise - percepção geográfica integrada, e; 4) Ganho de conhecimento - para ações sobre os espaços concretos (fundamentação para o planejamento e gestão territoriais locais).

Antes do exame de tais projetos, será apresentada a plataforma utilizada, apelidada de VICON (Sistema de VIgilância e CONtrole Web, do pacote SAGA/UFRJ - Sistema de Análise Geo-Ambiental da Universidade Federal do Rio de Janeiro), desenvolvida pelo Laboratório de Geoprocessamento da universidade mencionada.

\section{VICON.SAGA Web e Mobile}

A plataforma VICON.SAGA assemelha-se ao que vem sendo chamado por SIGWeb ou Cloud GIS. Ela é interoperável (Goodchild, 1997) e arquitetada para possibilitar um trabalho coletivo de ganho de conhecimento, ou seja, para além do acúmulo exaustivo de dados e da publicação de produtos temático-cartográficos acabados.

Trata-se de um repositório de projetos cujas características são: simultaneidade de operações; acolhimento de usuários; integração vertical e horizontal de dados e usuários; distribuição customizada de formulários semi-estruturados; importação e disseminação de dados, informações e metadados específicos; espacialização pontual por endereços ou coordenadas geográficas; importação de dados de outras plataformas (SIGs desktop, mobile, bancos de dados convencionais e redes sociais); guarda e utilização de arquivos multimídia; consulta de registros e atributos por respostas e/ou buffers; análise de densidades de pontos; simulação de rotas; exportação de relatórios gerenciais.

O VICON.SAGA demonstra-se uma solução de potencial gerenciador de projetos e decision-maker atuando nos âmbitos: corporativo (integrando diversos setores de trabalho), social (espacializando eventos e entidades componentes do cotidiano) ou híbrido (corporativo e social). No primeiro caso, possibilita-se o exercício do controle inerente às atividades de planejamento e gestão territoriais do governo e de empresas. No segundo, contribui-se com ações sociais e pedagógicas intrínsecas aos desenvolvimentos pessoal e comunitário. No caso do uso híbrido, projetam-se atividades de responsabilidade socioambiental, fundamentais para os preceitos da sustentabilidade.

Desta forma, há, a partir da inclusão digital, através da plataforma, que é gratuita, a inclusão social (da realidade percebida, vivida e representada) sob o referencial geográfico. A integração destas inclusões, apoiada no georreferenciamento, no conteúdo taxonômico e em tratamentos não-idiográficos gera a geoinclusão - uma condução ordenada dos problemas e tentativas de solução urbanos sobre uma base de conhecimento adequada à realidade (Xavier da Silva, 2011).

Cumpre notar, em termos de relações com desenvolvimentos recentes do processamento de dados, que a geoinclusão, assim, nutre e é nutrida pelo crowdthinking, na medida em que processa dados por tecnologia distribuída e gera informação para a construção e manutenção de projetos colaborativos. Estas características são encontradas em sua página (http://www.viconsaga.com.br/site/features), podem ser consultadas diretamente nos projetos abertos - também disponíveis (http://www.viconsaga.com.br/site/projects) - e serão finalmente demonstradas. Além disto, é minuciosamente descrita, fundamentada e demonstrada em Xavier da Silva (2008).

\section{Paisagismo}

Um projeto que merece destaque sempre que o tema for a geoinclusão está em andamento no Jardim Botânico da Cidade do Rio de Janeiro, no Brasil. Trata-se do "Paisagem Problema". Ele tem por finalidade o mapeamento de problemas percebidos tanto para manutenção do parque quanto para o conforto das espécies florais e dos visitantes.

Criou-se uma base de dados semi-estruturados que possibilitou a inclusão de registros ligados às condições de circulação, proteção florestal, manejo e reposição, de maneira aberta, sendo permitido o registro por parte de observadores voluntários e também corpo técnico-científico responsável em uma base georreferenciada totalmente compartilhada. Ou seja, construiu-se coletivamente o conhecimento para compreensão da realidade ambiental fazendo com que cada visitante se torne um potencial colaborador responsável pela saúde do parque e de seu entorno.

Como se pode inferir, o projeto surgiu com o intuito de operacionalizar o crowdsourcing para a manutenção do Jardim Botânico. Crowdsourcing é uma lógica de construção coletiva, que se diferencia do crowdthinking, pois este vai além (representa uma coletividade continuada e com vista à transformação da realidade). O crowdsourcing pode ter esta perspectiva, porém, conceitualmente, representa um esforço 
coletivo na construção de bases de dados distribuídas, sem necessariamente promover a informação.

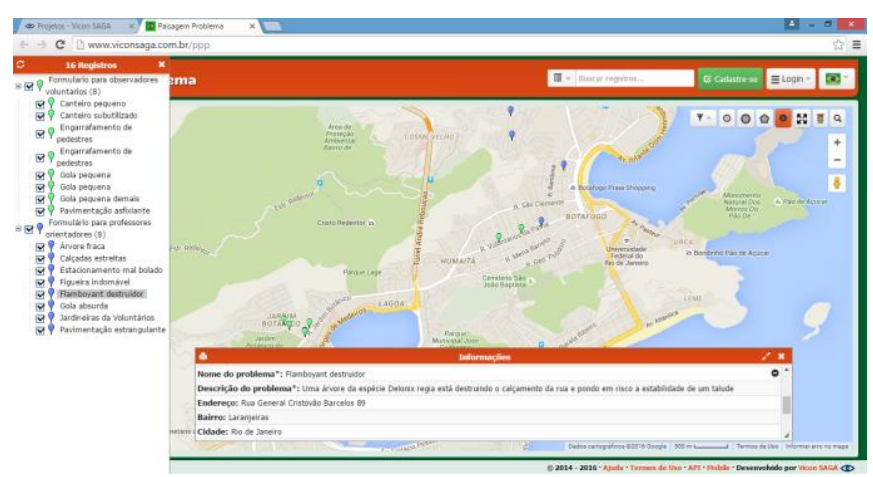

Figura 2: Inclusão de dados referentes ao Jardim Botânico do Rio de Janeiro e bairros vizinhos a partir de observadores voluntários e corpo técnico especializado. Crowdsourcing.

Foram identificados congestionamentos de pedestres e estrangulamentos de árvores com reflexos à destruição de calçadas e ameaças à estabilidade de taludes, já na fase de controle, devido à frequência de registros. Surge, a partir disto, proposições de projetos referentes à reavaliação das golas das árvores, acessibilidade dos passeios e manejo de flora.

\begin{tabular}{|l|}
\hline \multicolumn{1}{|c|}{ Informações } \\
\hline Nome do problema*: Pavimentação asfixiante \\
\hline Descrição do problema*: Árvores rodeadas de cimento, cujas raizes levantam o muro da praça \\
\hline Endereço: Rua Felício dos Santos \\
\hline Bairro: Santa Teresa \\
\hline Cidade: Rio de Janeiro, Brasil
\end{tabular}

Figura 3: Inclusão de detalhes referentes aos registros espaciais.

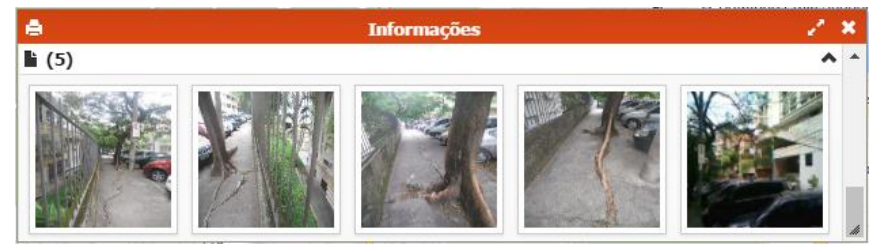

Figura 4: Inclusão de recursos multimídia para visualização do problema relatado.

Fica clara a possibilidade de se avaliar o ambiente para planejar ações de reparo e melhoria quanto ao conforto das espécies e dos atores envolvidos, promovendo um estreitamento entre a observação e a ação transformadora.

\section{Segurança}

Um projeto que testou a inclusão por output de dados, provenientes de redes sociais, deu-se no campo da Segurança Pública. Há uma opção de "importação de posts" no VICON que permite a busca e o georreferenciamento automático de postagens a partir de palavras-chave. Trata-se de um bom exemplo do uso híbrido do sistema, onde a rede social alimenta a base de dados gerando uma base de conhecimento compartilhado para que agentes de campo atuem sobre a realidade.
Em 2013, foi realizado experimento para aquisição de dados referentes à criminalidade na Cidade de Deus. Buscou-se "UPP" e "Cidade de Deus"; utilizou-se o filtro de ocorrências para "roubo"; materializou-se uma base de dados com 329 registros em um período quadrimestral.

Com a funcionalidade heatmap (figura 5), foram resultados:

1) Identificação de áreas com maior frequência de ocorrências e, portanto, maior possibilidade de repetição de fenômenos;

2) Possibilidade de mudança de rotas cotidianas da própria população;

3) Possibilidade de integração com dados demográficos e ambientais para compreensão dos fenômenos da violência urbana;

4) Possibilidade de integração com setores de inteligência para o planejamento de operações policiais como um serviço urbano (Vanagunas, 2007).

Muniz da Silva (2013) sugeriu dois usos a partir deste mesmo projeto. $O$ primeiro foi de se utilizar o heatmap para definição das áreas de direcionamento de viaturas, tendo, portanto, um projeto de curto prazo. Mas, com ênfase no planejamento de médio e longo prazos, sugeriu também georreferenciar para constratar com as ocorrências criminais todo recurso ou efetivo policiais disponíveis pelos bairros e cidades. Desta forma, tornou-se possível aplicar às unidades de policiamento um valor de oferta e demanda de segurança. Este valor, atribuído como em um sistema de notas, aplicando algoritmos disponíveis em SIGs desktop, possibilita o recorte das áreas de influência de cada unidade policial. Comparando as áreas de influência com as jurisdições territoriais de cada unidade, apoia-se a tomada de decisão quanto ao remanejamento de recursos para melhor atendimento às populações até então vulneráveis.

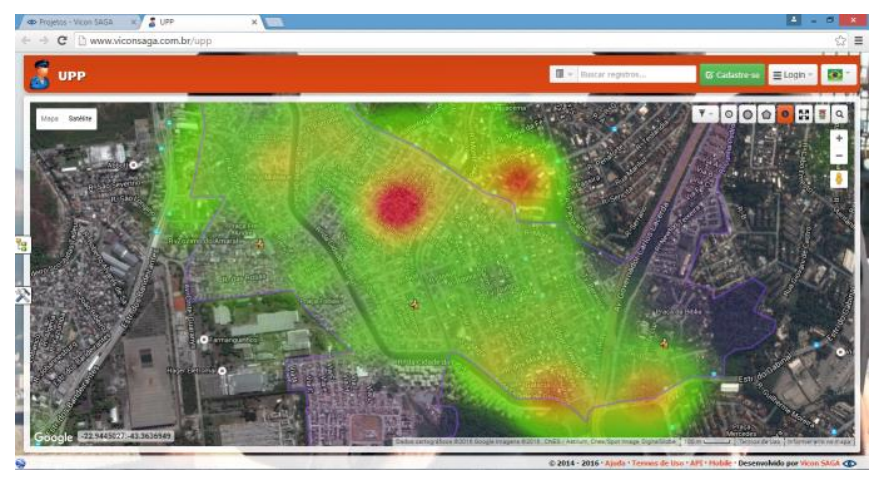

Figura 5: Funcionalidade heatmap para identifcação de áreas com necessidades de segurança. Aplicação do projeto UPP Cidade de Deus.

Este projeto pode ser melhor conhecido em Muniz da Silva (2013), onde há, inclusive, um passo-a-passo do projeto.

Assim como o heatmap, outras funcionalidades podem ser consultadas em "ferramentas de mapa", em qualquer projeto acessável através de /site/projects, assim como em /site/features - links já mencionados em página anterior. 


\section{Considerações finais}

O presente artigo destinou-se, principalmente, à visão conceitual e aplicada - sem uma apresentação de todas as características e funcionalidades do VICON e sem restrição aos usos e ideias que se desdobraram de cada projeto porque se vislumbra uma tendência tecnológica para as plataformas e dispositivos móveis, sem exclusividades e adestramentos. Isto ficou claro quando abordada a "Geografia das coisas" e os questionamentos iniciais para elaboração do conceito da geoinclusão. Para um exemplo em Segurança Pública, de uso civil de ferramentas similares ao VICON, pode-se citar o "Onde fui roubado" (http://www.ondefuiroubado.com.br/).

O caso do paisagismo bem demonstrou como a inclusão digital sob bases georreferenciadas abertas contribuem com a representação e consequentemente a percepção espacial dos grupos de pessoas sobre suas realidades para fins de melhorias ambientais.

Os projetos podem ser compostos de fases com diferentes níveis de participação, quando há necessidade posterior de intervenção de pessoal especializado. É possível programar permissões de carga de dados e criação de usuários para alimentação ordenada. O desejável para a cidadania plena é a abertura das bases de dados, pois a relação entre abertura e input de dados é diretamente proporcional às oportunidades empreendedoras de melhorias e solução de problemas de maneira cooperada. Para o gerenciamento dentro de uma corporação, no entanto, é um facilitador os diferentes níveis de permissão disponíveis pelo sistema. Disto deriva a classificação entre corporativo e/ou social.

O caso da segurança melhor demonstra o uso para a resiliência urbana por diversos atores. Há um output de dados gerados de maneira totalmente autêntica, por postagens em redes sociais, e que podem contribuir para 0 planejamento e a gestão territoriais por parte de agentes públicos, privados e também os cidadãos em seu dia-a-dia. A percepção da segurança na cidade, por exemplo, é cada vez mais trabalhada, havendo um prosseguimento lógico que passa pelo registro de ocorrências, a construção de bases representativas da realidade e possíveis relacionamentos propulsionados, principalmente, por posição geográfica. Podem ser cruzados nos projetos dados relacionados a projetos sociais, indicadores socioeconômicos, entre outros, fundamentais para a identificação de relações espaciais e, consequentemente, à definição de prioridades e prospecção de cenários possíveis.

Eis a operacionalização da geoinclusão, como enunciado desde 2008, e do crowdthinking, uma vez que se consolida a representação do conhecimento vivido em um sentido transformador.

O fato de se propiciar o engajamento entre pessoas e uma coesão social gera um trabalho colaborativo que, portanto, reduz custos, incentiva a ação comunitária, voluntária, e, assim, os desenvolvimentos pessoal e local. Os SIGs são fundamentais neste contexto, pois o território é quem integra todos os recursos, equipamentos e pessoas para estas ações. De maneira pedagógica, projetos são construídos nos mais diversos setores e é criado o sentimento de pertencimento e responsabilidade sobre o fazer ambiental.

Foram selecionados para este artigo os projetos em paisagismo e segurança pública, justamente, por se tratar de setores descentralizados, porém com possibilidade de integração para ações resilientes e a partir da autenticidade do viver os espaços.

Há, como perspectiva futura, no que tange o desenvolvimento do conceito, uma pesquisa de doutoramento sobre o uso da plataforma por organizações não-governamentais junto aos habitantes de uma favela da cidade do Rio de Janeiro. Estas são marcadas por uma suboferta de serviços e por uma multiplicidade de demandas e também ações criativas, formando um contexto de conflito.

\section{Agradecimentos}

Agradecemos à SIGraDI e, em especial, seu comitê científico pelas sugestões realizadas para a edição deste artigo, e à FAPERJ pelo financiamento de pesquisas.

\section{Referências bibliográficas}

Aronoff, S. (1989). Geographic information systems: a management perspective. Ottawa: WDL Publications.

Carroll, J. A. (2015). The colloborative mind: the acceleration of knowledge through crowd-thinking. Retirado de https://www.jimcarroll.com/2015/10/the-colloborative-mind-theacceleration-of-knowledge-through-crowd-thinking/.

Cereda Jr., A. (2015). Muito além da Internet das Coisas: a Geografia ds Coisas. Revista Conhecimento Prático: Geografia, 60(1), 30-31.

Goodchild, M.; Egenhofer, M.; Fegeas, R. (1997). Interoperating GISs. Santa Barbara: National Center for Geographic Information and Analysis.

Marino, T. B. (2015). Tratamento de informações geradas a partir de fontes de colaboração heterogêneas para apoio à resposta em emergências. (Tese de doutoramento). Programa de PósGraduação em Informática. Universidade Federal do Rio de Janeiro.

Muniz da Silva, L. O. (2013). Geoinclusão e segurança: o uso de geoprocessamento para o policiamento comunitário. (Monografia). Departamento de Geografia. Universidade Federal do Rio de Janeiro.

Vanagunas, S. (2007). Planejamento dos Serviços Policiais Urbanos. In: Jack R. Greene. (Org.). Administração do Trabalho Policial: Questões e Análises (pp. 43-60). São Paulo: EDUSP.

Xavier da Silva, J. (2008) Inclusão geográfica: um prosseguimento lógico entre as inclusões digital, social e geográfica. (Relatório de pesquisa). Departamento de Geografia. Universidade Federal do Rio de Janeiro.

Xavier da Silva, J. (2009). O que é Geoprocessamento. Revista do CREA-RJ, 79(1), 42-44.

Xavier da Silva, J. \& Marino, T. B. (2011). Inclusões: Digital, Social e Geográfica. In: Xavier da Silva, J. \& Zaidan, R. T. (Eds.). Geoprocessamento \& Meio Ambiente (pp. 17-34). Rio de Janeiro: Bertrand Brasil. 